\title{
Protecting unauthorized immigrant mothers improves their children's mental health
}

\author{
Jens Hainmueller, ${ }^{1,2,3 *}+$ Duncan Lawrence, ${ }^{2}+$ Linna Martén,,${ }^{2,4}+$ Bernard Black, ${ }^{5}$ Lucila Figueroa, ${ }^{2,6}$ Michael \\ Hotard, ${ }^{2}$ Tomás R. Jiménez, ${ }^{7}$ Fernando Mendoza, ${ }^{8}$ Maria I. Rodriguez, ${ }^{9}$ Jonas J. Swartz, ${ }^{9}$ David D. Laitin ${ }^{1,2}$ \\ ${ }^{1}$ Department of Political Science, Stanford University, Stanford, CA 94305, USA. ${ }^{2}$ Immigration Policy Lab, Stanford University, Stanford, CA 94305, USA. ${ }^{3}$ Graduate School \\ of Business, Stanford University, Stanford, CA 94305, USA. ${ }^{4}$ Uppsala Center for Labor Studies, Uppsala University, Uppsala 75120, Sweden. ${ }^{5}$ Pritzker Law School and \\ Kellogg School of Management, Northwestern University, Chicago, IL 60611, USA. ${ }^{6}$ Department of Politics, University of Virginia, Charlottesville, VA 22903 , USA. \\ ${ }^{7}$ Department of Sociology, Stanford University, Stanford, CA 94305, USA. ${ }^{8}$ Department of Pediatrics, Stanford University School of Medicine, Stanford, CA 94305, USA. \\ ${ }^{9}$ Department of Obstetrics and Gynecology, Oregon Health \& Science University, Portland, OR 97239, USA. \\ *Corresponding author. Email: jhain@stanford.edu
}

†These authors contributed equally to this work.

The United States is embroiled in a debate about whether to protect or deport its estimated 11 million unauthorized immigrants, but the fact that these immigrants are also parents to more than 4 million U.S.born children is often overlooked. We provide causal evidence of the impact of parents' unauthorized immigration status on the health of their U.S. citizen children. The Deferred Action for Childhood Arrivals (DACA) program granted temporary protection from deportation to more than 780,000 unauthorized immigrants. We used Medicaid claims data from Oregon and exploited the quasi-random assignment of DACA eligibility among mothers with birthdates close to the DACA age qualification cutoff. Mothers' DACA eligibility significantly decreased adjustment and anxiety disorder diagnoses among their children. Parents' unauthorized status is thus a substantial barrier to normal child development and perpetuates health inequalities through the intergenerational transmission of disadvantage.

There is an ongoing, heated debate about the fate of the estimated 11 million unauthorized immigrants living in the United States. One important and often overlooked issue in these policy debates is that unauthorized immigrants are also parents to more than 4 million children who are U.S. citizens by birth $(1,2)$. How are these children affected by the unauthorized status of their parents? Research has largely focused on the impacts of unauthorized status on the immigrants themselves (3), but we know much less about the potential intergenerational effects of this status on the well-being of their offspring (4).

A growing body of research has demonstrated links between parental immigration status and child development (5-10) and generated insights into how it might affect children's health. Children of unauthorized immigrant parents face challenges beyond low socioeconomic status, including parental anxiety, fear of separation, and acculturative stress. Parent-child separations can be harmful to children's health, economic security, and long-term development. Virtually all of these studies have been qualitative or correlational because of the difficulties in isolating the causal effects of parents' immigration status and collecting systematic data on large samples of unauthorized immigrants.

Families with unauthorized immigrant parents differ from families with authorized immigrant parents in many confounding characteristics (e.g., education, health care, and poverty) that might generate differences in child outcomes (11-13). This nonrandom selection implies that typical observational studies cannot isolate the causal effect of immigration status. Indeed, a recent consensus statement of the Society for Research on Adolescence (14) concludes that "Nonexperimental or quasiexperimental research with strong causal inference...has been lacking to date in studies of policies and practices related to unauthorized status."

The study of unauthorized status is further constrained by the difficulty of collecting systematic samples, because unauthorized immigrants are underrepresented in general population surveys (15). Moreover, questions about the unauthorized status of immigrants are typically avoided given concerns about confidentiality and reporting biases (16). Researchers therefore often have to resort to noisy proxies for unauthorized status, such as the identification of individuals as foreign-born, Hispanic, or Spanish-speaking (17, 18).

We provide causal evidence of the intergenerational impact of parental immigration status on children's health. We focus on the Deferred Action for Childhood Arrivals (DACA) program, which is one of the most extensive policies directed toward unauthorized immigrants in recent decades. The DACA program, announced in 2012 by President Obama, protects recipients from deportation by granting them a 2-year (renewable) deferred action status, while also allowing them 
to obtain temporary work authorization. More than 780,000 unauthorized immigrants so far have received deferred action through this program (19) (fig. S1). Although DACA recipients arrived in the United States as children, many are now adults and have become parents themselves. An estimated 200,000 children had parents who were eligible for DACA at the time the policy was announced (2). Although some studies have found that DACA recipients have higher rates of employment (20-22) and improved health outcomes $(23,24)$, the intergenerational effects of DACA are largely unknown.

To address the sampling problem, we used data from Emergency Medicaid, a government program that provides coverage for emergencies and labor and delivery services for low-income individuals who are not eligible for Medicaid. The program mainly serves unauthorized immigrants, but lawful permanent residents with less than 5 years of residency can also obtain coverage. Estimates from states such as California and North Carolina indicate that 90 to $99 \%$ of Emergency Medicaid recipients are unauthorized immigrants $(25,26)$. In addition, because U.S.-born children of unauthorized immigrants are U.S. citizens, they are eligible for full-scope Medicaid benefits and can be tracked with Medicaid claims data.

To overcome the causal identification problem, we applied a regression discontinuity (RD) design (27) that leverages the DACA eligibility criterion (28) stipulating that recipients must have been under age 31 as of 15 June 2012 . Hence, a person born on 16 June 1981 meets the DACA age eligibility requirement, whereas a person born on 14 June 1981 does not. The age eligibility criterion was announced when DACA was adopted on 15 June 2012. The Emergency Medicaid enrollment data include the mother's exact date of birth, and this permits us to leverage a quasi-experiment in which DACA eligibility is as good as randomly assigned for those born around the arbitrary birthdate cutoff. We do not observe whether mothers apply for DACA, but given that mothers who were born just before or after the DACA birthdate cutoff are similar in confounding characteristics, we can isolate the intention-to-treat effect of DACA eligibility on the health of their children. Prior studies provide evidence that $\mathrm{RD}$ designs that exploit arbitrary cutoff points in eligibility criteria are effective in replicating results from randomized experiments (29-31).

We drew on Medicaid claims data from Oregon to identify 5653 mothers born between 1980 and 1982 who were covered by Emergency Medicaid and gave birth to 8610 children during 2003 to 2015 . We then tracked the children's mental health outcomes by using their Medicaid claims. The children in our sample were born in Oregon and are therefore U.S. citizens by birth; $49 \%$ are female, $73 \%$ are Hispanic, and they were between 0 and 12 years old in 2015 (table S1 provides descriptive statistics).

Although parental DACA eligibility could affect a broad range of child health outcomes, we focused on the impacts on children's mental health. Because DACA offered the mothers immediate relief from the risk of deportation, maternal stress might have declined, and their children would no longer have had to fear being separated from them. Therefore, the children's mental well-being could have improved $(4,6)$. Moreover, examining mental health disorders that originate in childhood is important because they are associated with longterm health issues, low education, and welfare dependence, which generate considerable private and social costs (32-34).

We focused on disorders that result from external events, rather than genetic or physiological factors. We prespecified all outcomes and analyses, except when otherwise noted, in a preregistered analysis plan made available at the Evidence in Governance and Politics website under study ID 20170227AC. Our main child outcome is a broad measure of any diagnoses of adjustment disorder, acute stress disorder, or anxiety disorder, measured using all diagnoses in the International Classification of Diseases 9 (ICD-9) categories 309, 308, and 300 (35).

Adjustment disorder is a reaction to an identified stressor, leading to an inability to function normally. It is diagnosed on the basis of symptoms of anxiety, depressed mood, and conduct disturbances and often results in considerable impairment in important areas of functioning, such as social activities, school performance, and sleep $(36,37)$. Acute stress disorder can be a precursor to a diagnosis of a more lasting posttraumatic stress disorder (included in the ICD-9 category 309 , adjustment disorder). It is characterized by symptoms or behaviors similar to those that arise from exposure to a traumatic or stressful event, but acute stress disorders cannot (by definition) last longer than 1 month (36). Because stress disorder and adjustment disorder are related, we prespecified both as a combined outcome measure of adjustment disorder. Anxiety disorders are characterized by excessive fear, anxiety, and related behavioral disturbances that can lead to substantial distress or impairment. An external stressor might not be clearly identified, and anxiety disorders can be caused by environmental, genetic, or physiological factors (36).

These mental health disorders in childhood are associated with considerable developmental, psychosocial, and psychopathological complications for children and their families (32). For the children in our sample who were diagnosed with adjustment disorder, acute stress disorder, or anxiety disorder, the first diagnoses occurred on average at 6.7 years of age with a standard deviation of 2.6 years (tables S1 and S2 provide descriptive statistics). Details about the measures, sample, design, and statistical analysis can be found in the materials and methods section of the supplementary materials. 
Figure 1 illustrates the main finding and quasi-experimental nature of the RD design. The percent of children diagnosed with adjustment or anxiety disorders during the post-DACA period (2013 to 2015) dropped by about 4.5 percentage points $(P=0.037$; local linear regression $)$ at the birthdate cutoff where mothers become eligible for DACA. This reduction, from 7.8 to $3.3 \%$, provides evidence that mothers' DACA eligibility sharply improved their children's mental health.

The causal logic of the RD design is based on the idea that the DACA birthdate cutoff is an arbitrary date, and, therefore, children of ineligible mothers born just before the birthdate cutoff should be similar in all respects, including in possible confounding characteristics, to children of DACA-eligible mothers born just after the cutoff. This continuity assumption was corroborated by a series of checks where we tested for discontinuities in pre-DACA background characteristics at the DACA birthdate cutoff. The results (Fig. 1, bottom left) demonstrate that there was no discernible difference in the prevalence of disorder diagnoses at the same cutoff date for the pre-DACA period (2003 to quarter 2, 2012). The difference in diagnosis rates at the cutoff was an insignificant 0.4 percentage points $(P=0.817$; local linear regression). Figure 1 , bottom right, shows the distribution of $P$ values from similar checks where we tested for discontinuities in other background covariates at the birthdate cutoff, such as the children's ethnicity, race, year of birth, and pre-DACA health care utilization (tables S3 and S4). The distribution of $P$ values is consistent with the uniform distribution that we would expect for balance checks in a randomized experiment, indicating that there were no systematic discontinuities in the covariates at the birthdate cutoff. Furthermore, density tests for manipulation of mothers' birthdates revealed no evidence of sorting around the threshold (fig. S2). All tests suggested that our RD design can isolate the causal effects of mothers' DACA eligibility at the birthdate cutoff.

Figure 2 shows the point estimates and confidence intervals for the $\mathrm{RD}$ estimates of the intention-to-treat effects of mothers' DACA eligibility on the children's mental health outcomes, for the combined measure and its separate components (tables S5 and S6 and fig. S3). The estimates are based on prespecified standard local linear regression models fitted to trimmed samples including only children whose mothers' birthdates were within the adaptive mean squared error optimal bandwidths around the birthdate cutoff (38).

We found that mothers' eligibility for DACA protection led to a significant improvement in their children's mental health. Specifically, mothers' DACA eligibility reduced adjustment and anxiety disorder diagnoses in their children by 4.3 percentage points $(P=0.023)$ from a baseline rate of $7.9 \%$ among children of ineligible mothers at the threshold. This represents more than a $50 \%$ drop in the rate of these disorders, albeit with a wide $95 \%$ confidence interval (CI) for the magnitude of the estimated effect, ranging from 0.6 to 7.9 percentage points. When we looked only at adjustment disorders, which are disorders attributable to an identifiable external stressor, the estimated reduction was 4.4 percentage points $(P=0.013 ; 95 \% \mathrm{CI}, 0.9$ to 7.8$)$. There was also a reduction in anxiety disorders, which is a more heterogeneous category of mental illness, but it was insignificant at conventional levels ( $P=0.153 ; 95 \% \mathrm{CI},-0.6$ to 4.1$)$. Lastly, we found that for the same sample of children, before the DACA program, there were no discernible differences in these mental health diagnoses at the cutoff (Fig. 2, right).

We conducted several checks that supported the robustness of the results, such as varying the bandwidths (fig. S4), using alternative estimation procedures (fig. S5 and table S7), removing children born in the post-DACA period (fig. S6 and table S8), redefining the post-DACA period to include quarters 3 and 4 of 2012 (fig. S7 and table S9), and using alternative codings of the mental health outcomes based on the Diagnostic and Statistical Manual of Mental Disorders (fig. S8 and table S10; not prespecified). A non-prespecified subgroup analysis (fig. S9) suggested that the effect of mothers' DACA eligibility was concentrated among the older children in our sample (ages 6 to 12; table S12), with no discernible effect among younger children (ages 0 to 5; table S11); younger children are generally much less likely to receive mental health diagnoses. We also conducted a non-prespecified subgroup analysis by gender (fig. S10 and tables S13 and S14) and found that the effect of mothers' DACA eligibility on adjustment disorders was slightly more pronounced among male children, but the effect for males was not statistically significantly different from that for females $(P=0.209$; local linear regression).

We also confirmed that there were no discernible differences in diagnoses at the same birthdate cutoff among children of mothers who were covered by standard Medicaid at the time that they gave birth (fig. S11 and table S15). These mothers should not be affected by DACA eligibility, given that standard Medicaid in Oregon is open only to low-income U.S. citizens and long-term lawful permanent residents. This check again underscores that in the absence of changes in DACA eligibility, there is no evidence of confounders associated with having a mother who is born just before or after the cutoff date that could explain the observed post-DACA difference in child mental health outcomes.

Because health care utilization could be affected by immigration status (9), we also checked for the possibility that the drop in diagnoses reflects a DACA-induced change in health care visits, which could affect the probability of detection of mental health disorders. We found no support for this. Moth- 
ers' DACA eligibility had no discernible impact on their children's health care utilization during the post-DACA period, as measured either by the total number of visits, the number of emergency room (ER) and urgent care visits, or the number of outpatient visits (fig. S12 and table S16). Consistent with this, in a non-prespecified analysis, we also found that the effects of mothers' DACA eligibility on child mental health were similar when we restricted the sample to children who had at least one health care visit in the post-DACA period (fig. S13 and table S17).

Our results provide causal evidence supporting the theory that parental unauthorized immigration status has important intergenerational effects on the well-being and development of children in immigrant families $(4,6)$. Protecting unauthorized immigrants from deportation led to immediate and sizable improvements in the mental health of their U.S. citizen children. This suggests that parents' unauthorized status is a substantial stressor that stymies normal child development and perpetuates health inequalities by transferring parental disadvantages to children.

Our findings have important implications for immigration and health care policy. As decision-makers evaluate whether to maintain, cancel, or expand the DACA program, our results suggest that a broader consideration is needed, one that goes beyond the impacts for recipients alone and takes into account the intergenerational consequences of deferred action for the health of unauthorized immigrants' children, most of whom are U.S. citizens (2). Early childhood exposure to stress and adversity does not only cause poor health and impaired development in the short term; the issues can also persist into adulthood. Anxiety and psychosocial stress are identified as risk factors for depression, substance abuse, cardiovascular diseases, and obesity $(32,34$, 39,40 ). Treatment of mental disorders also carries considerable economic costs to society. They account for the highest total health care expenditures of all children's medical conditions (41) and are associated with poor long-term outcomes for school performance and welfare reliance $(33,42)$. By reducing mental health problems, deferred action has important multiplier effects through improving the future prospects of the children of unauthorized immigrants.

Our results imply that expanding deferred action to the millions of unauthorized immigrant parents who do not meet the current DACA eligibility criteria could further promote the health and well-being of this next generation of American citizens. Moreover, it is reasonable to expect that permanent legal status or a pathway to citizenship would have an equal, if not greater, effect on improving children's health.

Our study also has implications for health policy research. Unauthorized immigration is an important policy issue, but researchers have struggled to generate a reliable evidence base. Although we recognize the limitations of evaluating health outcome data from one state, our sampling strategy of using Emergency Medicaid mothers and Medicaid children provides an effective way to overcome some of the challenges in collecting systematic data from the unauthorized population. This approach opens the door for future studies to examine the impacts of an array of local, state, and federal policies that affect unauthorized immigrant parents and that may have health consequences for their children.

\section{REFERENCES AND NOTES}

1. J. S. Passel, D. Cohn, J. M. Krogstad, A. Gonzalez-Barrera, As Growth Stalls, Unauthorized Immigrant Population Becomes More Settled (Pew Research Center's Hispanic Trends Project, 2014); www.pewhispanic.org/files/2014/09/2014-09-03_Unauthorized-Final.pdf.

2. R. Capps, M. Fix, J. Zong, "A profile of U.S. children with unauthorized immigrant parents" (Migration Policy Institute, 2016).

3. P. M. Orrenius, M. Zavodny, The economic consequences of amnesty for unauthorized immigrants. Cato J. 32, 85 (2012).

4. F. D. Bean, S. K. Brown, J. D. Bachmeier, Parents Without Papers: The Progress and Pitfalls of Mexican American Integration (Russell Sage Foundation, 2015).

5. S. R. Potochnick, K. M. Perreira, Depression and anxiety among first-generation immigrant Latino youth: Key correlates and implications for future research. J. Nerv. Ment. Dis. 198, 470-477 (2010). doi:10.1097/NMD.0b013e3181e4ce24 Medline

6. H. Yoshikawa, A. Kalil, The effects of parental undocumented status on the developmental contexts of young children in immigrant families. Child Dev. Perspect. 5, 291-297 (2011). doi:10.1111/j.1750-8606.2011.00204.x

7. F. D. Bean, M. A. Leach, S. K. Brown, J. D. Bachmeier, J. R. Hipp, The educational legacy of unauthorized migration: Comparisons across U.S.-immigrant groups in how parents' status affects their offspring. Int. Migr. Rev. 45, 348-385 (2011). doi:10.1111/j.1747-7379.2011.00851.x Medline

8. C. Suárez-Orozco, H. Yoshikawa, R. Teranishi, M. Suárez-Orozco, Growing up in the shadows: The developmental implications of unauthorized status. Harv. Educ. Rev. 81, 438-473 (2011). doi:10.17763/haer.81.3.g23×203763783m75

9. K. Yun, E. Fuentes-Afflick, L. A. Curry, H. M. Krumholz, M. M. Desai, Parental immigration status is associated with children's health care utilization: Findings from the 2003 new immigrant survey of US legal permanent residents. Matern. Child Health J. 17, 1913-1921 (2013). doi:10.1007/s10995-012-1217-2 Medline

10. T. M. Caballero, L. R. DeCamp, R. E. Platt, H. Shah, S. B. Johnson, E. M. S. Sibinga, S. Polk, Addressing the mental health needs of Latino children in immigrant families. Clin. Pediatr. 6, 648-658 (2017). Medline

11. F. L. Rivera-Batiz, Undocumented workers in the labor market: An analysis of the earnings of legal and illegal Mexican immigrants in the United States. J. Popul. Econ. 12, 91-116 (1999). doi:10.1007/s001480050092 Medline

12. J. P. Smith, Immigrants and the labor market. J. Labor Econ. 24, 203-233 (2006). doi:10.1086/499971

13. K. P. Derose, J. J. Escarce, N. Lurie, Immigrants and health care: Sources of vulnerability. Health Aff. 26, 1258-1268 (2007). doi:10.1377/hlthaff.26.5.1258 Medline

14. H. Yoshikawa, C. Suárez-Orozco, R. G. Gonzales, Unauthorized status and youth development in the United States: Consensus statement of the society for research on adolescence. J. Res. Adolesc. 27, 4-19 (2017). Medline

15. U.S. Government Accountability Office, U.S. Labor Force Statistics: Illustrative Simulations of the Likely Effects of Underrepresenting Unauthorized Residents (Tech. Rep. GA0-10-99, U.S. Government Accountability Office, 2009).

16. National Academies of Sciences, Engineering, and Medicine, The Integration of Immigrants into American Society, M. C. Waters and M. G. Pineau, Eds. (National Academies Press, 2015).

17. J. Van Hook, J. D. Bachmeier, D. L. Coffman, O. Harel, Can we spin straw into gold? An evaluation of immigrant legal status imputation approaches. Demography 52 , 329-354 (2015). doi:10.1007/s13524-014-0358-x Medline

18. R. S. Oropesa, N. S. Landale, M. M. Hillemeier, Family legal status and health: Measurement dilemmas in studies of Mexican-origin children. Soc. Sci. Med. 138 57-67 (2015). doi:10.1016/j.socscimed.2015.05.038 Medline 
19. U.S. Citizenship and Immigration Services, Data Set: Form I-821D Deferred Action for Childhood Arrivals (Department of Homeland Security, U.S. Citizenship and Immigration Services, 2012-2016); www.uscis.gov/tools/reportsstudies/immigration-forms-data/data-set-form-i-821d-deferred-actionchildhood-arrivals.

20. R. G. Gonzales, V. Terriquez, S. P. Ruszczyk, Becoming DACAmented: Assessing the short-term benefits of Deferred Action for Childhood Arrivals (DACA). Am. Behav. Sci. 58, 1852-1872 (2014). doi:10.1177/0002764214550288

21. N. G. Pope, The effects of DACAmentation: The impact of Deferred Action for Childhood Arrivals on unauthorized immigrants. J. Public Econ. 143, 98-114 (2016). doi:10.1016/j.jpubeco.2016.08.014

22. C. Amuedo-Dorantes, F. Antman, Schooling and labor market effects of temporary authorization: Evidence from DACA. J. Popul. Econ. 30, 339-373 (2017). doi:10.1007/s00148-016-0606-z Medline

23. A. S. Venkataramani, S. J. Shah, R. O'Brien, I. Kawachi, A. C. Tsai, Health consequences of the US Deferred Action for Childhood Arrivals (DACA) immigration programme: a quasi-experimental study. Lancet Public Health 2 , e175-e181 (2017)

24. C. Patler, W. Laster Pirtle, From undocumented to lawfully present: Do changes to legal status impact psychological wellbeing among latino immigrant young adults? Soc. Sci. Med. 10.1016/j.socscimed.2017.03.009 (2017). doi:10.1016/i.socscimed.2017.03.009 Medline

25. C. A. DuBard, M. W. Massing, Trends in emergency Medicaid expenditures for recent and undocumented immigrants. JAMA 297, 1085-1092 (2007). doi:10.1001/jama.297.10.1085 Medline

26. C. H. C. Foundation, Medi-Cal Facts and Figures: A Program Transforms (California Health Care Foundation, 2013)

27. G. W. Imbens, T. Lemieux, Regression discontinuity designs: A guide to practice. J. Econom. 142, 615-635 (2008). doi:10.1016/i.jeconom.2007.05.001

28. The other main DACA eligibility criteria include entry to the United States under the age of 16, continuous residence from 15 June 2007 to the present, entry without inspection or falling out of lawful visa status before 15 June 2012, physical presence in the United States on 15 June 2012, current enrollment in school or a high school or GED (General Education Development) degree, and no major criminal convictions.

29. H. Buddelmeyer, E. Skoufias, An Evaluation of the Performance of Regression Discontinuity Design on PROGRESA (Policy Research Working Paper, World Bank, 2004).

30. T. D. Cook, W. R. Shadish, V. C. Wong, Three conditions under which experiments and observational studies produce comparable causal estimates: New findings from within-study comparisons. J. Policy Anal. Manage. 27, 724-750 (2008). doi:10.1002/pam.20375

31. R. Berk, G. Barnes, L. Ahlman, E. Kurtz, When second best is good enough: A comparison between a true experiment and a regression discontinuity quasiexperiment. J. Exp. Criminol. 6, 191-208 (2010). doi:10.1007/s11292-010-9095$\underline{3}$

32. K. Beesdo, S. Knappe, D. S. Pine, Anxiety and anxiety disorders in children and adolescents: Developmental issues and implications for DSM-V. Psychiatr. Clin. North Am. 32, 483-524 (2009). doi:10.1016/j.psc.2009.06.002 Medline

33. J. Currie, M. Stabile, P. Manivong, L. L. Roos, Child health and young adult outcomes. J. Hum. Resour. 45, 517-548 (2010). doi:10.3368/ihr.45.3.517

34. J. P. Shonkoff, A. S. Garner, B. S. Siegel, M. I. Dobbins, M. F. Earls, A. S. Garner, L. McGuinn, J. Pascoe, D. L. Wood, The lifelong effects of early childhood adversity and toxic stress. Pediatrics 129, e232-e246 (2012). doi:10.1542/peds.2011-2663 Medline

35. When we use the terms adjustment disorder, acute stress disorder, or anxiety disorder, we refer to all the diagnoses included under the ICD-9 categories 300, 308, and 309, respectively. These categories include several subcategories of diagnoses. For example, we observed 13 subcategories under category 309 (adjustment reaction), such as 309.0 (adjustment disorder, depressed mood), 309.21 (separation anxiety disorder), 309.24 (adjustment disorder, anxiety), 309.3 (adjustment disorder, disturbance of conduct), and 309.81 (posttraumatic stress disorder). Table S2 in the supplementary materials provides a list of all subcategories, as well as descriptive statistics.

36. A. F. Schatzberg, C. DeBattista, Diagnostic and Statistical Manual of Mental
Disorders (American Psychiatric Association, ed. 5, 2015).

37. L. C. Garfunkel, J. Kaczorowski, C. Christy, Pediatric Clinical Advisor: Instant Diagnosis and Treatment (Elsevier Health Sciences, 2007).

38. S. Calonico, M. D. Cattaneo, R. Titiunik, Robust nonparametric confidence intervals for regression-discontinuity designs. Econometrica 82, 2295-2326 (2014). doi:10.3982/ECTA11757

39. A. Thapar, S. Collishaw, D. S. Pine, A. K. Thapar, Depression in adolescence. Lancet 379, 1056-1067 (2012). doi:10.1016/S0140-6736(11)60871-4 Medline

40. K. R. Merikangas, J. P. He, M. Burstein, S. A. Swanson, S. Avenevoli, L. Cui, C. Benjet, K. Georgiades, J. Swendsen, Lifetime prevalence of mental disorders in U.S. adolescents: Results from the National Comorbidity Survey ReplicationAdolescent Supplement (NCS-A). J. Am. Acad. Child Adolesc. Psychiatry 49, 980 989 (2010). doi:10.1016/j.jaac.2010.05.017 Medline

41. A. Soni, The Five Most Costly Children's Conditions, 2011: Estimates for the US Civilian Noninstitutionalized Children, Ages 0-17 (Statistical Brief \#434, Agency for Healthcare Research and Quality, 2014).

42. J. Currie, D. Almond, Human capital development before age five. Handb. Labor Econ. 4, 1315 (2011)

43. J. McCrary, Manipulation of the running variable in the regression discontinuity design: A density test. J. Econom. 142, 698-714 (2008). doi:10.1016/j.jeconom.2007.05.005

44. M. D. Cattaneo, M. Jansson, X. Ma, "Simple local regression distribution estimators with an application to manipulation testing" (Working Paper, University of Michigan, 2016).

\section{ACKNOWLEDGMENTS}

This research was funded by a grant from the Russell Sage Foundation (grant no. 9316-12). We also acknowledge funding from the Ford Foundation for operational support of the Stanford Immigration Policy Lab. For helpful advice, we thank K. Bansak, V. G. Carrion, A. Hainmueller, and J. Wang. Replication code is available through Harvard Dataverse (https://dataverse.harvard.edu/dataset.xhtml?persistentld=doi:10.7910/DVN/ 8EEDAP). A preregistered analysis plan is available at the Evidence and Governance in Politics website under study ID 20170227AC

(http://egap.org/design-registrations). The analysis plan is also reprinted in the supplementary materials. The Institutional Review Boards at Stanford University (protocol 40907) and Oregon Health \& Science University (protocol 15633) approved this research.

\section{SUPPLEMENTARY MATERIALS}

www.sciencemag.org/cgi/content/full/science.aan5893/DC1

Materials and Methods

Supplementary Text

Figs. S1 to S13

Tables S1 to S17

References $(43,44)$

Preregistered Analysis Plan

5 May 2017; accepted 1 August 2017

Published online 31 August 2017

10.1126/science.aan5893 


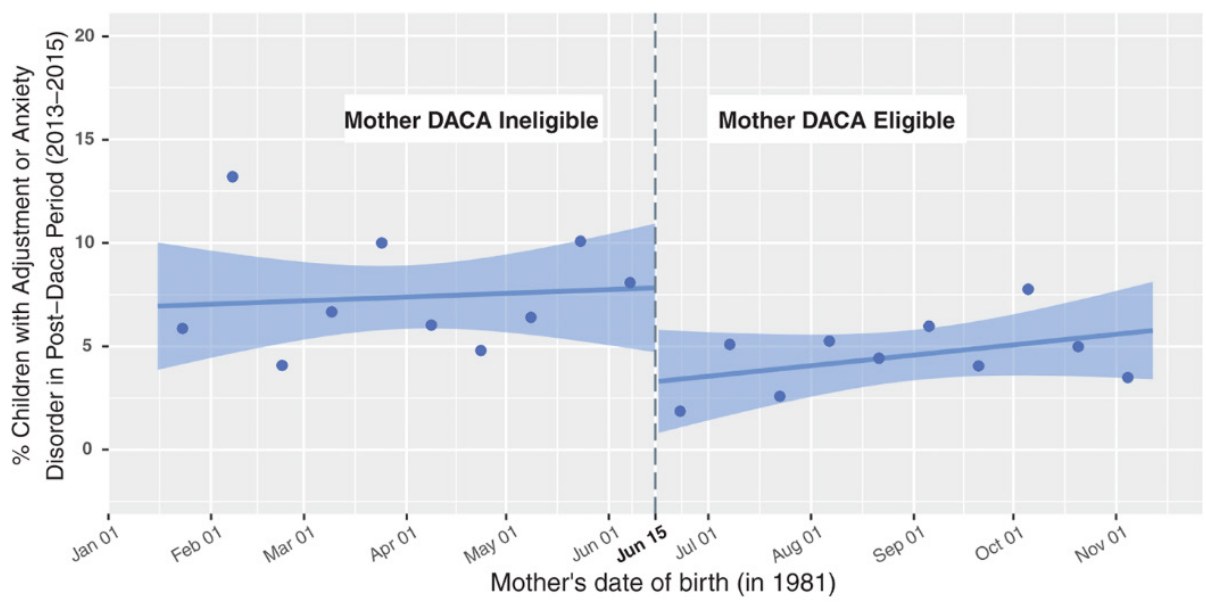

Fig. 1. Results from applying the regression discontinuity design. (Top) In the post-DACA period (2013 to 2015), children of DACAeligible mothers (born after 15 June 1981) experienced markedly lower rates of diagnosed adjustment and/or anxiety disorders than children of ineligible mothers (born before 15 June 1981). Lines are average diagnosis rates (with 95\% confidence bands) from local linear regressions fitted to the sample of children whose mothers' birthdates
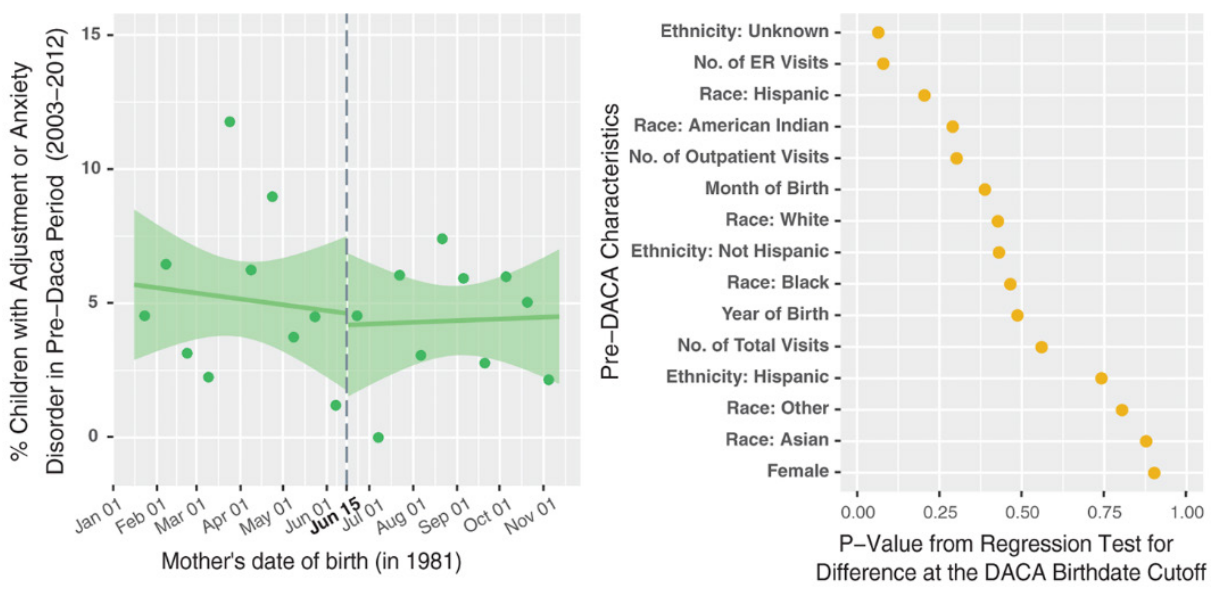
were within \pm 150 days of the DACA eligibility cutoff ( $n=2260)$, and circles are average diagnosis rates within each 15-day birthdate interval. (Bottom left) There was no such difference in children's diagnosis rates in the pre-DACA period (2003 to 2012). (Bottom right) There was no statistical evidence for discontinuities in other background characteristics that might confound the comparison at the DACA birthdate eligibility cutoff. 


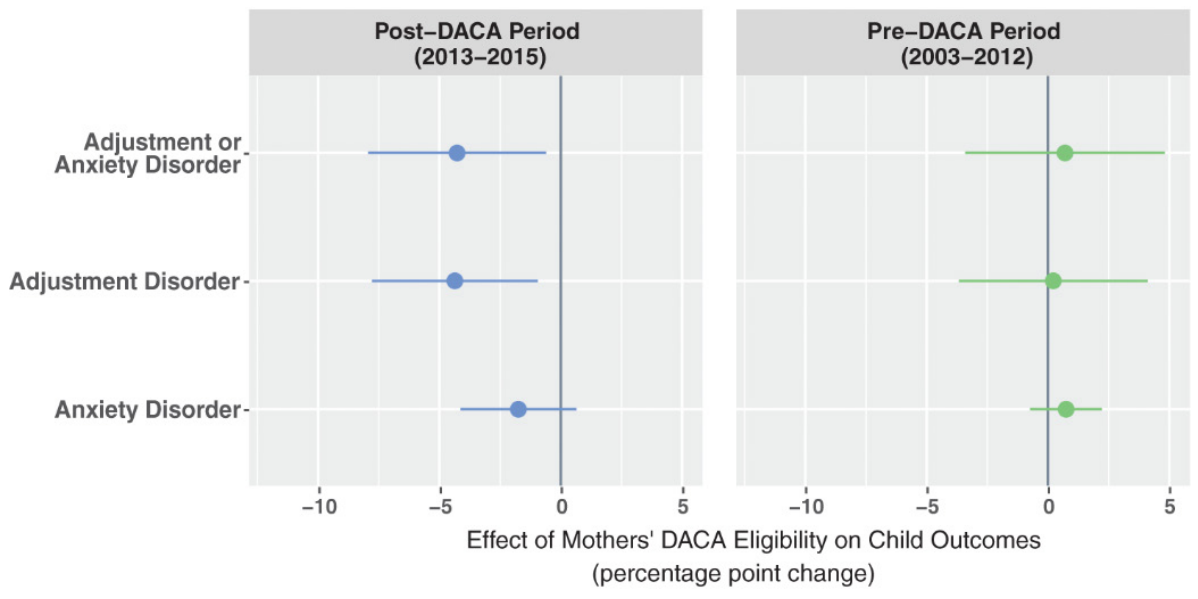

Fig. 2. Effect of mothers' DACA eligibility on their children's mental health. (Left) Mothers' DACA eligibility reduced child mental health disorders in the post-DACA period. (Right) There were no systematic, preexisting differences in the pre-DACA period. Circles with lines represent effect estimates with 95\% confidence intervals from the regression discontinuity design, based on local linear regressions fitted to samples of children whose mothers' birthdates were within a symmetric bandwidth of days around the DACA eligibility cutoff. The size of the bandwidth was determined by an adaptive bandwidth selection algorithm for each outcome. The bandwidths and sample sizes for the three outcomes in the post-DACA period (top to bottom) are \pm 199 days around the cutoff $(n=$ 3039 children), \pm 180 days ( $n=2741$ ), and \pm 132 days $(n=2002)$; for the preDACA period (top to bottom), the bandwidths and sample sizes are \pm 108 days $(n=1325), \pm 109$ days $(n=1338)$, and \pm 211 days $(n=2745)$. 